\title{
Chemical Sensors in Environmental Chemistry - Monitoring Air and liquid Phase
}

\author{
F. L. Dickert, U. Latif, G. Mustafa, S. Yaqub, B. Najafi, P. A. Lieberzeit \\ Department of Analytical Chemistry, University of Vienna \\ Waehringer Str. 38, A-1090 Vienna, Austria \\ I. Heeren, Robert Bosch GmbH, Applied Research, Stuttgart
}

\section{Introduction:}

A large number of toxic and hazardous compounds originate from the industries, automobiles and degradation processes ${ }^{1}$. Moreover, some hazardous pollutants called herbicides are widely used to control certain weeds in agriculture field. Environment pollution can be reduced by using ethanol as fuel in place of mineral oils.

In composting, volatile sulfur compounds produced, which are poisonous and give foul-smelling. The halitosis, bad odor of feces and flatus is due to presence of sulfur volatiles and detection of these VSCs might give valuable informations about some systematic diseases and metabolic disorders. Therefore, a thiol detection chemosensor is very important from biological, chemical, clinical, pharmacological and environmental point of view. Sulfur containing compounds in the environment can be detected by using the Pearson approach of "Hard and Soft acid base concept". By using this idea metal sulfide materials were used for the analysis of thiols. Detection of thiols will take place via affinity attractions between soft sulfur atom of the compound and soft metal of sensitive layer.

In all over the world especially in the developing countries, atrazine and related triazine herbicides are widely used to control weeds in agricultural field. These herbicides and their derivatives are hazardous pollutants and remain in soils, natural waters and environmental domain for long time ${ }^{2}$. Their presence in natural reservoir can cause serious health risks like birth defects, cancer, weight loss, and disorder in hormone functionality ${ }^{3}$. Some techniques which are commonly used for the detection of herbicides are gas and liquid chromatography ${ }^{4,5}$ in combination with mass spectrometry, and other electrochemical methods ${ }^{6}$. These ways of detecting thiols are although precise but time consuming, costly and required skilled personals for their operation. Besides these techniques, chemosensors are more generalized and universally-adaptive devices for online measurements.

Molecularly imprinted polymers (MIPs) in combination with quartz crystal microbalances (QCMs) have proved a useful approach to design artificial receptors for online monitoring of analytes ${ }^{7,8}$. The straightforward method to produce MIP is combination of functional monomers and template in a covalent or non-covalent way ${ }^{9,10}$. The template may be the analyte itself or any other suitable compound may be added to pattern the sensitive layer. Self-organization of monomers around template leads to highly selective molecular imprints in the nanometer or sub-nanometer range. Removing the template from the polymer leaves behind the interaction sites suitable for analyte incorporation. These structured polymers can be applied to any transducer such as quartz crystal microbalance (QCM) or interdigital transducer (IDT), for the reversible inclusion of the analytes. QCMs are used for gravimetric analysis and their theoretical detection limit is up to pico-gram level ${ }^{11}$. Interdigital transducers ${ }^{12}$ (IDTs) are finger or comblike periodic pattern of electrodes on glass substrate, used to investigate the change in electrical properties of sensitive layer coated on it.

The environment pollution can also be reduced by adding ethanol as fuel in place of mineral oils because: petroleum reservoirs are going down and regeneration of the fuel without further addition of carbon dioxide in the atmosphere. In this case the resistive measurements were performed using salvation effects for the detection of different concentrations of ethanol in fuel in reversible manner.

\section{Sensitive coating for thiol detection:}

In designing recognition material which specifically and reversibly interacts with the sulfur containing compounds in the presence of corresponding oxygen and nitrogen containing materials is a tedious task. Thiols show strong interactions with soft metals like silver or gold but these bindings are irreversible. So, these metals cannot be used as sensitive coatings because of regeneration problem but these soft metals have given us an idea that soft materials can be used as recognition coatings on transducer. Thus, the Pearson proposed "hard and soft acid-base concept" leads us for choosing a 
sensitive material for thiols. By using this idea, a soft metal sulfide i.e. MoS ${ }_{2}$ nanoparticles was synthesized by our group for the detection of thiols ${ }^{13}$. In contrast to sensitive layer, nanoparticles of MoS $_{2}$ were synthesized, which offers increased interaction area and sensitivities with favorable response times.

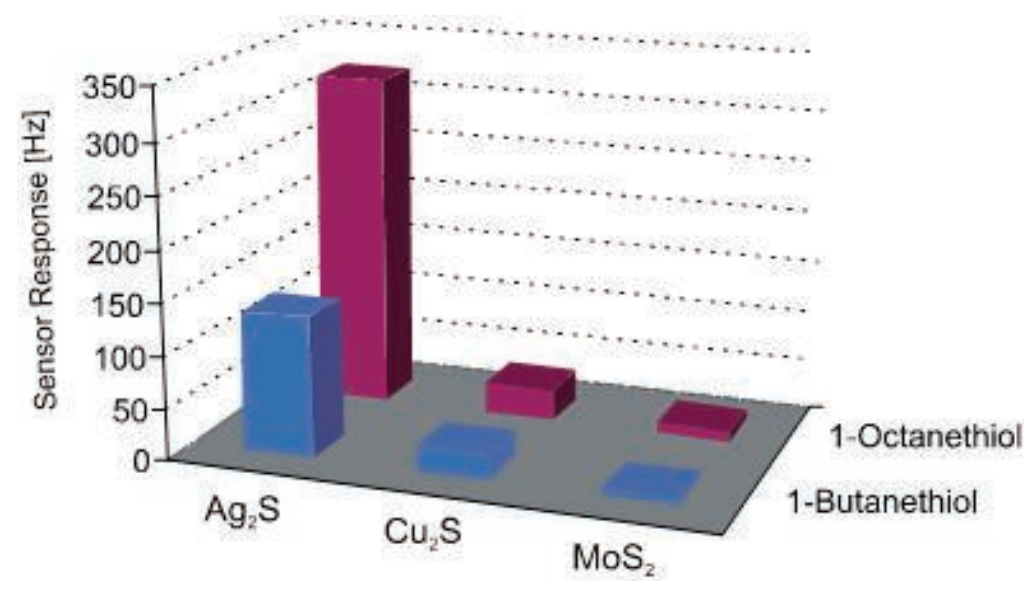

Figure 1: Sensor response of molybdenum sulfide, copper sulfide, and silver sulfide nanoparticles against 1-Butanethiol and 1-Octanethiol

We extended our approach to other more soft metals than molybdenum such as copper and silver to achieve maximum sensitivity. These metals i.e. especially silver proved themselves as promising candidates to design a recognition material for the detection of toxic and foul-smelling class of organic compounds. Softness of metal and nanoparticles size are of crucial importance especially in case of thiol detecting sensor, a decrease in particles size and increase in softness of metal molybdenum < copper < silver both enhance the sensitivity of sensor. Soft metal sulfide nanoparticles approach has proven to be highly suitable for generating recognition sensor materials. Silver sulfide nanoparticles have been proved a promising candidate for thiols detection. The sensitivity and selectivity comparison of $\mathrm{MoS}_{2}, \mathrm{Cu}_{2} \mathrm{~S}$ and $\mathrm{Ag}_{2} \mathrm{~S}$ nanoparticles against different analytes is illustrated is figure 1. Brownish-black nanoparticles of $\mathrm{MoS}_{2}$ were produced by reacting molybdenum hexacarbonyl with sulfur under nitrogen environment ${ }^{14}$. Nanoparticles of $\mathrm{Cu}_{2} \mathrm{~S}$ were produced by mixing the aqueous solution of copper sulfate with sodium thiosulfate and keep the mixture unattended at ambient conditions for a week. Black-green precipitates of copper sulfide were separated, washed and dried at $60^{\circ} \mathrm{C}$ for 24 hours ${ }^{15}$. $\mathrm{Ag}_{2} \mathrm{~S}$ nanoparticles were synthesized by passing the $\mathrm{H}_{2} \mathrm{~S}$ gas through the alcoholic solution of silver nitrate. In this way silver sulfide nanoparticles were precipitated out, which were collected, dried and used for measurement ${ }^{16}$.

\section{Detection of herbicides with MIPs:}

Atrazine is an organic compound consisting of triazine ring, is widely used to stop grassy weeds in major crops. Structural formulas of atrazine and its structural analogous are shown in figure 2. All these compounds contain triazine ring in common.<smiles>CCNc1nc(Cl)nc(NC(C)C)n1</smiles>

(a)<smiles>CC(C)Nc1nc(Cl)nc(NC(C)C)n1</smiles>

(b)<smiles>Nc1nc(N)nc(Cl)n1</smiles>

(c)

Figure 2: Structural formulas of (a) Atrazine, (b) Propazine, and (c) Desethyl-desisopropyl atrazine

In designing imprinted polymer for herbicides, functionalized monomers like acrylate/methacrylate were used. To introduce geometrical features for reversible inclusion of analytes, it was templated with atrazine. Adjusting acid to cross-linker ratio as well as introducing acrylate ester as copolymer resulted in optimal non-covalent interaction with the hydrophobic core of the atrazine molecule and the amino groups. A non-imprinted polymer material was also synthesized in the same way but without adding template. Molecularly imprinted polymer is coated on one channel of $10-\mathrm{MHz}$ dual-electrode QCM and on 
other channel non-imprinted polymer material was coated for differential measurements. Sensor effects of different concentration of atrazine in aqueous solution were obtained with excellent signal to noise ratio. The imprinted material incorporates considerable amount of atrazine from aqueous solution than non-imprinted material as shown in figure 3. Sensor response of about $80 \mathrm{~Hz}$ was obtained while exposing the $0.4 \mathrm{mg} / \mathrm{L}$ concentration of atrazine in aqueous solution towards imprinted polymer layer; whereas, in case of non-imprinted layer, the response was only $10 \mathrm{~Hz}$. The imprinted material further incorporates considerable amount of atrazine at lower concentrations as compared to reference material and depicts a detection limit of $100 \mathrm{ppb}$. These concentration dependent frequency shifts on sensor shows substantial imprinting effects of the material. These measurements show fast response time and excellent reversibility of the sensor.

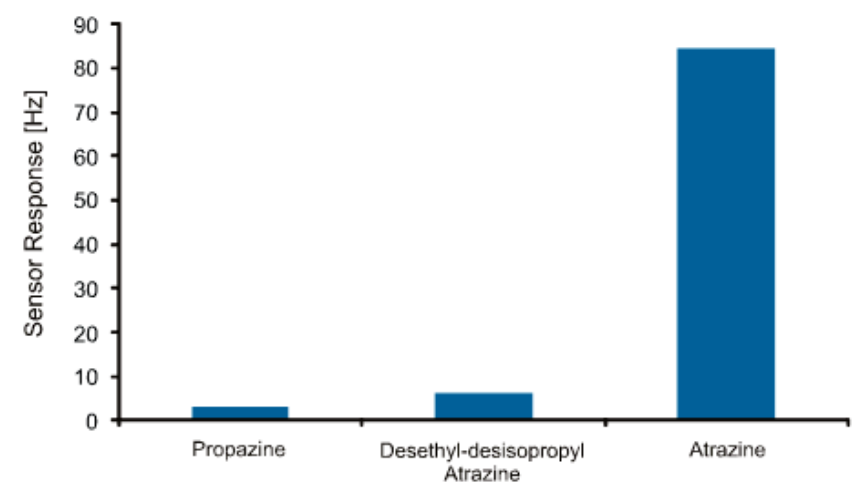

Figure 4: Cross selectivity of molecularly imprinted synthetic antibodies for Atrazine in comparison to propazine and desethyl-desisopropyl atrazine.

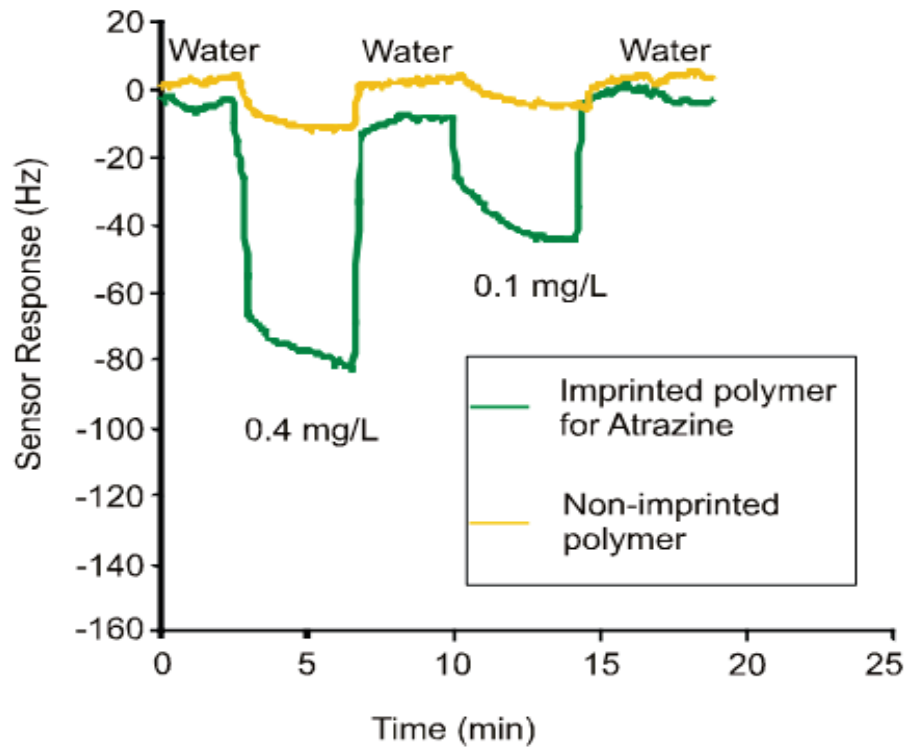

Figure 3: Sensor response of imprinted and nonimprinted polymer of acrylate/methacrylate different concentrations of Atrazine in water at $28^{\circ} \mathrm{C}$

\section{Fuel Monitoring:}

Furthermore, the hydrocarbons in fuel can be characterized. Sol-gel materials enable us to distinguish between isomeric hydrocarbons and to correlate the QCM sensor responses with the "WienerIndex" describing stereochemistry. Imprinting polymers with n-paraffins and branched hydrocarbons will give selective responses to isomers. Additionally, we focus on characterizing the composition of fuel to detect main components and ingredients. To improve the environmental conditions, bio-alcohol is added to fuel and a sensor was developed which allow a continuous detection of this alcohol. In principle, this can be done by uncoated capacitors since alcohol has an appreciable larger dielectric constant than hydrocarbons. The cross-selectivity to other ingredients: oxidation products as acetic acid and combustions modifiers as added ethers cannot be guaranteed by this simple procedure. Thus, a sensitive layer was applied and combined with QCMs and IDTs. Measurements can be most easily performed according to changes in conductivity in less polar fuel. As sensor materials, crosslinked acrylic acids were used to form a network of polyelectrolytes and exposed to different concentrations of alcohol in fuel as 
shown in Fig. 5. Appreciable changes in resistance of sensitive layer were observed by adding bio-alcohol to fuel. Lowering of resistance is due to separation of ion pairs $\mathrm{M}^{+} \mathrm{X}^{-}$in mobile ions by solvation effects. Thus, the ion pair which does not contribute in conductance will turn into mobile ions. The range of observed effects can be varied by using different ionic networks. The basicity of anions drastically can be varied and cationic counter ions with different diameter can be used to tune the desired effects.

\section{Conclusion:}

Nanoparticles of $\mathrm{MoS}_{2}, \mathrm{Cu}_{2} \mathrm{~S}$, and $\mathrm{Ag}_{2} \mathrm{~S}$ have been synthesized, which can interact reversibly and specifically with sulfur containing compounds. Thus, these materials can be used

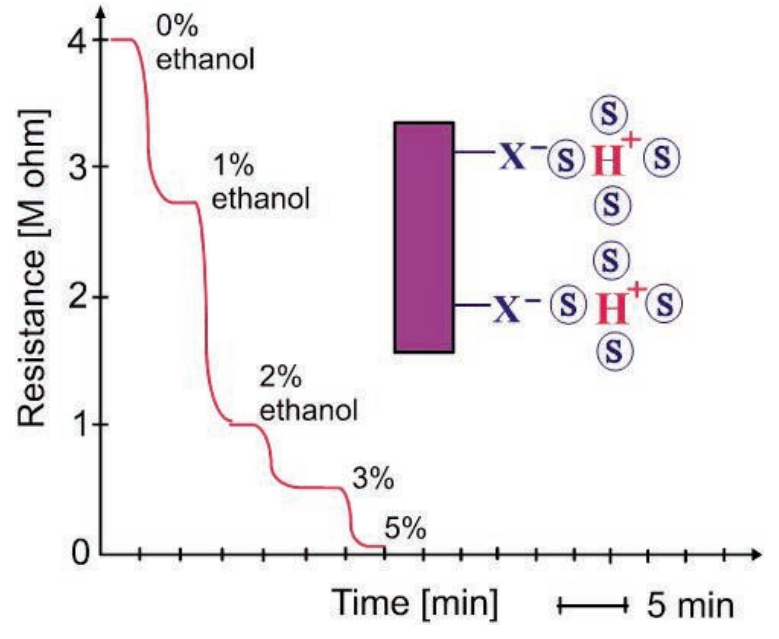

Figure 5: Measurement of the resistance as a function of different \%ages of ethanol in fuel for the detection of toxic and smelly class of compounds. So, more is the softness of the metal molybdenum<copper<silver greater will be the sensitivity of layer. Molecularly imprinted polymers have been synthesized from acrylate/methacrylate for the sensing of herbicides. Studies revealed that imprinted layers show excellent sensitivity and detection limit of $100 \mathrm{ppb}$ towards atrazine. Moreover, cross selectivity measurement depicts that appreciable higher sensor responses were obtained for atrazine in comparison to its structural analogues by using MIPs as sensitive layers. Highly crosslinked acrylic acid was used as a sensitive layer on interdigital transducer for the characterization of fuel. Remarkable changes in resistance were observed while exposing different concentrations of ethanol in fuel, due to salvation effects.

A. Tangerman, Journal of Chromatography B 877 (2009), 3366-3377.

N. D. Jablonowski, S. Köppchen, D. Hofmann, A. Schäffer, P. Burauel, Environmental Pollution 157 (2009), 2126-2131.

A. Hipólito-Moreno, M. E. León-González, L. V. Pérez-Arribas, L. M. Polo-Díez, Analytica Chimica Acta 362 (1998), 187-192.

M. C. Bruzzoniti, C. Sarzanini, G. Costantino, M. Fungi, Analytica Chimica Acta 578 (2006), 241249.

M. K. Ross, N. M. Filipov, Analytical Biochemistry 351 (2006), 161-173.

N. Maleki, G. Absalan, A. Safavi, E. Farjami, Analytica Chimica Acta 581 (2007), 37-41.

F. L. Dickert, P. Lieberzeit, S. G. Miarecka, K. J. Mann, O. Hayden, C. Palfinger, Biosens.

Bioelectron. 20 (2004), 1040-1044.

F. L. Dickert, P. Lieberzeit, S. Gazda-Miarecka, K. Halikias, K-J. Mann Sens. Actuators. B 100 (2004), 112-116.

K. Mosbach, O. Ramstrom, Nat. Biotechnol. 14 (1996), 163-170.

M. J. Whitcombe, M. E. Rodriguez, P. Villar, E. N. Vulfson, J. Am. Chem. Soc. 117 (1995), 71057111.

A. Mirmohseni, H. Abdollahi, K. Rostamizadeh Sens. Actuators B 121 (2007), 365-371.

U. Trebbe, M. Niggemann, K. Cammann, G. C. Fiaccabrino, M. Koudelka-Hep, S. Dzyadevich, O. Shulga, Fresenius J. Anal. Chem. 371 (2001), 734-739.

P. A. Lieberzeit , A. Afzal, A. Rehman, F. L. Dickert, Sensors and Actuators B 127 (2007), 132136.

D. Dominique, B. Stephane, L.C. Claude, Journal of Materials Chemistry 12 (2002), 2430-2432.

Y. Ni, F. Wang, H. Liu, Y. Liang, G. Yin, J. Hong, X. Ma, Z. Xu, Inorganic Chemistry Communications 6 (2003) 1406-1408.

S. Shukla, S. Seal, S. R. Mishra, Journal of Sol-Gel Science and Technology 23 (2002),151-164. 